\title{
Minimally Invasive Hallux Valgus Surgery. A Systematic Review.
}

\section{Edvin Selmani ${ }^{1 *}$, Fatmir Brahimi ${ }^{1}$, Leard Duraj ${ }^{1}$, Valbona Selmani ${ }^{1}$, Gjergji Syko ${ }^{1}$, Igli Karanca ${ }^{1}$}

Received: 25 March 2018; Accepted: 30 June 2018; Published online: 20 July 2018 DOI: https://doi.org/10.32391/ajtes.v2i2.5

\begin{abstract}
Background; This systematic review aims to illustrate the published results of "minimally invasive" procedures for correction of hallux valgus. Based on former systematic reviews on that topic, the literature search was organised by two independent investigators. MEDLINE was systematically searched for available studies. The keywords used were "hallux valgus", "bunion", "percutaneous surgery", "minimally invasive surgery", "arthroscopy", "Bosch" and "SERI". Studies were assessed using the level of evidence rating. A total of 21 papers were included in this review. These studies described a total of 1,750 patients with 2,195 instances of percutaneous, minimally invasive or arthroscopic hallux valgus surgery. Clinical reports of results after minimally invasive hallux valgus surgery at meetings are common. Published results in peer-reviewed journals are less common and the majority of papers are level IV studies according to the level of evidence ratings. We found one level II and three level III studies. Reported complications seem to be less than one may see in one's own clinical practice. This possible bias may be related to the fact that most studies are published by centres performing primarily minimally invasive hallux valgus surgery.

Keywords: Minimally invasive procedures, hallux valgus, bunion, percutaneous surgery, arthroscopy
\end{abstract}

*Corresponding Author: Edvin Selmani

$\equiv$ Email; selmaniedvin@gmail.com

${ }^{1}$ Service of Orthopedics and Trauma No. 2, University Hospital of Trauma, Tirana, Albania 


\section{Introduction}

The earliest reports of surgical hallux valgus correction date back to Gernet in 1836. Procedures for resection of parts of the first metatarsophalangeal (MTP) joint by the colleagues Hueter, Mayo [1], Keller [2] and Brandes [3] became the most popular.

Since these techniques were proven to fail, correctional metatarsal osteotomies replaced them; again our ancestors in the nineteenth century had already thought the same way. The first reports date back to Reverdin [4] who described in 1881 a subcapital closing wedge osteotomy for the correction of hallux valgus deformity. It then became popular as the Hohmann [5] osteotomy. From the beginning of the use of osteotomies for the treatment of hallux valgus deformities, surgeons distinguished between distal and proximal osteotomies. While Hohmann [5], Wilson [6], Mitchell [7] and chevron [8] osteotomies were representatives of the distal osteotomies, Loison [9], Balacescu [10], Ludloff [11], Trott [12] and crescentic Mann osteotomies [13] were representatives of the proximal osteotomies. One might think that after more 160 years of facing the problem of hallux valgus surgery the ideal treatment should have been found. In 1931 Peabody [14] thought that he had found it. He stated that all of his patients were happy and there were no complications. Unfortunately, he was wrong and surgeons are still seeking wisdom. Helal in 1981 [15] counted more than 150 different techniques and the number has continued to increase.

At least a minimum consensus among surgeons has been established. Minor and moderate deformities [rated by intermetatarsal angle (IMA) and hallux valgus angle (HVA)] may be treated by distal osteotomies, while more severe deformities are best treated by proximal metatarsal osteotomies, which have been mathematically proven to give the best corrections.

Ferrari [16] published a systematic review in 2002 and concluded that there was no compelling evidence for an advantage of any of these techniques over any other particular type of surgery.

The role of minimally invasive techniques Minimally invasive surgery has gained popularity in all fields of orthopaedic and trauma surgery. The theoretical advantages are potentially decreased recovery and rehabilitation times, reduced surgical time and less stress to the patient.

One may distinguish between minimal incision surgery, percutaneous surgery and arthroscopic surgery [17]. Arthroscopic hallux valgus surgery is certainly not a standard procedure [18]. It is demanding, time-consuming and carries the potential risk of nerve injury $[19,20]$.

Early reports of percutaneous hallux valgus surgery date back to the 1940s [21] in the USA, where podiatrists were trying to circumvent the restrictive laws regarding surgery for podiatric physicians. Early power equipment for minimal incision metatarsal osteotomies was developed in the 1960s.

Peter Bösch modified the popular Kramer osteotomy after a conversation with the podiatrist O.T. New. His so-called subcapital osteotomy (SCOT) technique [22] became the origin of all percutaneous techniques of hallux valgus surgery that were later invented. The disadvantage of the percutaneous technique is the fact that surgery is performed without direct visualisation of the different tissue layers and intraoperative fluoroscopy is mandatory. The bony procedures are performed using a high-speed power bur which entails the disadvantage of bone loss which leads to shortening of the metatarsal. 
The minimal incision hallux valgus surgery is a technique where the smallest necessary incision to create the osteotomy is performed by using a power saw blade. These techniques are generally not performed under fluoroscopy [21].

The current question is whether it is justifiable to use percutaneous or minimal incision hallux valgus surgery based on the actual medical literature.

\section{Literature search and data extraction}

Based on the systemic reviews by Mafulli et al. [17] and Roukis [21] the literature search was organised by two independent investigators. MEDLINE was systematically searched for available studies. The keywords used were "hallux valgus", "bunion", "percutaneous surgery", "minimally invasive surgery", "arthroscopy", "Bosch" and "SERI". Once the relevant articles had been retrieved, they were hand-searched for further references fitting the search pattern. Only articles published in peerreviewed journals were included. If a reference could not be obtained it was excluded from consideration. Studies were assessed using the level of evidence rating introduced by the Journal of Bone and Joint Surgery (American Volume) in 2003 [23].

\section{Results}

A total of 21 papers were included in this review. These studies described a total of 1,750 patients with 2,195 instances of percutaneous, minimally invasive or arthroscopic hallux valgus surgery. Almost all studies were level IV studies, three studies could be rated as level III and one study as level II (Table 1).

Bösch et al. [24] and Markowski et al. [25] published in 1990 and 1991 the preliminary results with the SCOT (percutaneous) technique in 45 patients and 64 feet. The average follow-up was 16 months (range eight to 32 months). The range of motion decreased from preoperatively $66.5 \%$ of the patients presenting a range of motion between 70 and $110^{\circ}$ to $35 \%$ at final follow-up. There were also $9 \%$ pin track infections.

In 2000 Bösch et al. [22] reported much better results in a seven to ten year follow-up study. Of the 98 feet the HVA was corrected from preoperatively $36^{\circ}$ (range $14-54^{\circ}$ ) to $19^{\circ}$ (range 7$40^{\circ}$ ), and the IMA was corrected from $13^{\circ}$ (range 6$18^{\circ}$ ) to $10^{\circ}$ (range $3-18^{\circ}$ ) at follow-up. Complications included four deep infections and four cases of delayed bone healing. Portaluri [26] in 2000 published a series of the Bösch technique in 143 patients with 182 operated feet. The radio- logical results were almost identical to those Bösch et al. [22] reported. Only two patients had a pin track infection, two had early accidental pin removal and eight had reported superficial infections.

Magnan et al. [27] in 2005 reported on 118 consecutive percutaneous distal metatarsal osteotomies at an average of 35.9 months. Significant correction of all radiological parameters was reported. In $61 \%$ either plantar or dorsal displacement of the capital fragment was noted. Only one deep infection at the osteotomy site and superficial skin irritation by the Kirschner wire were reported. First MTP joint range of motion of less than $30^{\circ}$ was seen in seven patients $(6.8 \%)$. In 2013 lannò et al. [28] presented 72 patients ( 85 feet) treated with the Bösch method with an average follow-up of 73.3 months. HVA, IMA and sesamoid position were statistically significantly corrected ( $p<0.1$ for all). Complications included three cases of avascular necrosis (AVN) of the metatarsal head, malunion in four feet and two cases of skin irritation due to the Kirschner wire. 
A multicentre study of the distal percutaneous metatarsal osteotomy according to Isham was presented by Bauer et al. [29]. A total of 189 feet were prospectively followed with a mean follow-up of 13 months (12-24 months). The osteotomies were percutaneously performed and no internal fixation was used. The American Orthopaedic Foot and Ankle Society (AOFAS) score improved from preoperatively 52 points on average to 93 points at follow-up. The median range of motion decreased from $90^{\circ}$ preoperatively to $75^{\circ}$ which is an average of $17 \%$ loss of motion. Neither nonunion, AVN nor malunion of the capital fragment was reported. The authors state that their technique requires a learning phase before being able to produce reliable acceptable results.

In a single-institution study Bauer [30] reported his own results with the percutaneous Isham technique. A total of 104 cases in 82 feet were followed for a median of two years. AOFAS score, HVA and sesamoid position were significantly improved. Due to the philosophy of the Isham technique correction of the IMA is only possible to a limited extent. Besides two cases of limited range of motion, two cases of complex regional pain syndrome (CRPS) and six cases of distal metatarsal articular angle (DMAA) overcorrection, no complications were reported.

Radwan and Mansour [31] performed a comparative study between 31 percutaneous distal metatarsal osteotomies (Bösch technique) and 33 distal chevron osteotomies. The operative time results revealed on average seven minutes less surgical time with the percutaneous surgery. There was no difference in bony healing and radiographic parameters. The mean range of motion was almost identical in both groups. They further described that limited dorsal range of motion occurred more often in the chevron group. According to the report there was no nonunion or malunion after the percutaneous distal metatarsal osteotomy. Roth et al. [32] presented a retrospective comparative study of the percutaneous (Bösch) and the standard Hohmann techniques. There were 88 percutaneous and 36 open osteotomies performed. Radiological parameters revealed similar results in both groups. The complication rate with $15 \%$ infection, $10 \%$ nonunion and $6 \%$ CRPS in the percutaneous group was much higher than in the open group.

In 2009 Maffulli et al. [33] compared a series of 36 Scarf osteotomies with a series of 36 Bösch osteotomies. The mean operative time for the Bösch technique was significantly less then for the Scarf osteotomy (19 vs 42). Radiographic evaluation revealed similar results with both techniques.

Range of motion at follow-up was not reported in this series. There were three pin track infections after the Bösch technique.

Giannini et al. [34] performed a prospective comparative study of the SERI (simple, effective, rapid, inexpensive) and Scarf osteotomies. Twenty patients with bilateral hallux valgus deformities underwent a Scarf on one side and a SERI osteotomy on the other side. All patients underwent clinical and radiological follow-up at two and seven years. The results of the radiological correction between the two groups were similar in respect of HVA, IMA and DMAA. In this study there were no reported intraoperative or post-operative complications in either group. All osteotomies healed properly. Most interesting were the reported surgical times. A very short surgical time of 17 minutes on average with the Scarf osteotomy was even topped by the average surgical time of three minutes with the SERI.

Enan et al. [35] evaluated the early results of the minimally invasive hallux valgus correction using the minimal incision Hohmann-type technique. At a 
mean follow-up of 21 months (12-36 months) 24 patients with 36 operated feet, a preoperative IMA $\leq 18^{\circ}(9-18)$ and an HVA of more than 17 and less than $40^{\circ}$ formed the study group. Without knowing the pre-operative AOFAS score, the score at final follow-up was 91.1 points. In only $44.4 \%$, the position of the metatarsal head was found without plantar (47.3\%) or dorsal displacement (88.3\%). Shortening on an average of $2.2 \pm 2.8 \mathrm{~mm}$ was noted. The HVA was corrected from an average of $27.7^{\circ}(18-37)$ to $14.6^{\circ}(8-24)$ and the IMA from 11.2 ! (10-18) to $5.8^{\circ}(4-12)$. As complications only three feet with mild inflammatory skin reactions around the outlet of the K-wire were observed.

\section{Discussion}

Post-operative range of motion and stiffness after the percutaneous or minimally invasive Hohmanntype osteotomy $[27,35]$ is a matter for discussion. Despite the fact that a K-wire is inserted for six weeks beside the joint capsule and the first MTP joint has no range of motion for six weeks the reported numbers of stiffness are low [27, 35]. In their original paper Markowski et al. [25] reported a decrease of range of motion in $31 \%$ of patients. Radwan and Mansour [31] compared the mean post- operative range of motion after the percutaneous Bösch method and distal chevron. Although the first technique includes fixation of the first MTP joint for six weeks while the distal chevron allows immediate range of motion and as described range of motion exercises after four weeks, the presented results showed almost identical mean post-operative range of motion at follow-up. Unfortunately most studies do not report the postoperative loss in range of motion [26, 32]. A potential disadvantage of the percutaneous and minimally invasive distal metatarsal osteotomies according to the Hohmann technique is the plantar or dorsal malalignment. While Bösch et al. [22], Scala and Vendettuoli [36], Sun et al. [37], Giannini et al. [34] and Radwan and Mansour [31] did not report metatarsal head position at follow-up, Magnan et al. [27] reported $61 \%$, Enan et al. [35] $55.6 \%$ and lannò et al. [28] $4.7 \%$ dorsal or plantar malalignment.

Most studies did not mention the incidence of malunion or nonunion after percutaneous or minimally invasive distal metatarsal osteotomies. Kadakia et al. [38] planned a randomised prospective study. Due to their early complications with the minimally invasive technique they stopped the study. One may argue that despite the fact that the authors were extremely experienced foot and ankle surgeons, their experience in minimally invasive hallux valgus surgery was minimal and they started the study with the first patient they planned for surgery.

We found three comparative studies of minimally invasive distal metatarsal osteotomies vs open hallux valgus operations. All studies revealed similar corrections of all radio- graphic parameters. Maffulli et al. [33] reported a highly significant shorter surgical time with the percutaneous technique (19 vs 42 min), while in Radwan and Mansour's [31] study only a minimal but statistically significant difference ( 50 vs 58 minutes, $p=0.015$ ) was reported. In Giannini et al.'s [34] study an extremely short surgical time was reported. Duration of surgery is an issue in hallux valgus correction; nevertheless, this should not be the only argument when choosing one procedure over the other.

Reported complications vary among the studies. While Giannini et al. [34] reported no complications at all in their comparative series, other authors noted complications. Bösch reported in 1990 pin track infection in $9.5 \%$ and Roth et al. [32] in $15 \%$. Bösch et al. [22] stated in 2000 that the infection 
rate decreased after completing the learning curve. Bauer et al. [29], Portaluri [26], Scala and Vendettuoli [36] and lannò et al. [28] reported few incidences of pin track infections.

Correction of radiographically measured deformity is the objective aim of hallux valgus surgery. This can certainly be achieved using minimally invasive hallux valgus surgery. Comparisons of minimally invasive and open techniques revealed similar results of both techniques [17, 31, 32, 34, 44, 45].

\section{Conclusion}

Clinical reports of results after minimally invasive hallux valgus surgeries at meetings are common. Published results in peer-reviewed journals are less common and the majority of papers are level IV studies according to the level of evidence ratings. We found one level II and three level III studies. Reported complications seem to be less than one may see in one's own clinical practice. This possible bias may be related to the fact that most studies are published by centres performing primarily minimally invasive hallux valgus surgery. Correction of deformity is certainly similar to open hallux valgus surgery. One definite advantage is the reduced time of surgery, and the disadvantage especially with the Bösch technique is the potentially reduced range of motion after surgery. Studies of higher levels of evidence with larger numbers of cases should be conducted.

\section{References}

1. Mayo CH (1908) The surgical treatment of bunion. Ann Surg 48: 3002

2. Keller WL (1904) The surgical treatment of bunions and hallux valgus. N Y Med J

80:741-742
3. Brandes M (1924) Zur operation therapie des hallux valgus. Zentralbl Chir 56:243244

4. Reverdin J (1881) De la deviation en dehors du gros orl (hallux valgus) et son traitement chirurgical. Trans Int Med Congr 2:408-412

5. Hohmann G (1921) Symptomatische oder physiologische Behandlung des Hallux valgus. Münchner Med Wschr 33:10421045

6. Wilson DW (1980) Treatment of hallux valgus and bunions. Br J Hosp Med 24:548549

7. Mitchell C, Fleming J, Allen R, Glenney C, Sanford G (1958) Osteotomybunionectomy for hallux valgus. J Bone Joint Surg Am 40:41-60

8. Austin DW, Leventen EO (1981) A new osteotomy for hallux valgus: a horizontally directed " $\mathrm{V}$ " displacement osteotomy of the metatarsal head for hallux valgus and primus varus. Clin Orthop 157:25-30

9. Loison M (1901) Note sur le traitment chirurgical du hallux valgus d'apres l'etude radiographique de la deformation. Bull Soc Chir Paris 27:528-531

10. Balacescu J (1903) Un caz de hallux valgus simetric. Rev Chir (Rumenia) 7:128-135

11. Ludloff K (1918) Die Beseitigung des Hallux valgus durch die schräge planta-dorsale Osteotomie des Metatarsus I. Arch Klin Chir 110:364-387

12. Trott AW (1972) Hallux valgus in the adolescent. Instr Course Lect 21:262-268

13. Mann RA (1990) Distal soft tissue procedure and proximal metatarsal osteotomy for correction of hallux valgus deformity. Orthopedics 13: 1013-1018 
14. Peabody CW (1931) The surgical cure of hallux valgus. J Bone Joint Surg 13:273-282

15. Helal B (1981) Surgery for adolescent hallux valgus. Clin Orthop 157:50-63

16. Ferrari J (2002) Hallux valgus (bunions). Clin Evid 8:1103-1112

17. Maffulli N, Longo UG, Marinozzi A, Denaro $V$ (2011) Hallux valgus: effectiveness and safety of minimally invasive surgery. A systematic review. Br Med Bull 97:149-167

18. Lui TH (2008) First metatarsophalangeal joint arthroscopy in patients with hallux valgus. Arthroscopy 24:1122-1129

19. Lui TH, Chan KB, Chow HT, Ma CM, Chan PK, Ngai WK (2008) Arthroscopy-assisted correction of hallux valgus deformity. Arthros- copy 24:875-880

20. Lui TH, Chan KB, Chan LK (2010) Endoscopic distal soft-tissue release in the treatment of hallux valgus: a cadaveric study. Arthroscopy 26:1111-1116

21. Roukis TS (2009) Percutaneous and minimum incision metatarsal osteotomies: a systematic review. J Foot Ankle Surg 48:380-387

22. Bösch P, Wanke S, Legenstein R (2000) Hallux valgus correction by the method of Bösch: a new technique with a seven-toten-year follow-up. Foot Ankle Clin 5:485498, v-vi

23. Wright JG, Swiontkowski MF, Heckman JD (2003) Introducing levels of evidence to the journal. J Bone Joint Surg Am 85-A:1-3

24. Bösch P, Markowski H, Rannicher V (1990) Technik und erste Ergebnisse der subcutanen distalen Metatarsale-IOsteotomie. Orthop Praxis 26:51-56

25. Markowski HP, Bosch P, Rannicher V (1991) Surgical technique and preliminary results of percutaneous neck osteotomy of the first meta- tarsal for hallux valgus. Foot 2:93-98

26. Portaluri $M(2000)$ Hallux valgus correction by the method of Bösch: a clinical evaluation. Foot Ankle Clin 5:499-511

27. Magnan B, Pezzè L, Rossi N, Bartolozzi P (2005) Percutaneous distal metatarsal osteotomy for correction of hallux valgus. J Bone Joint Surg Am 87:1191-1199

28. Iannò B, Familiari F, De Gori M, Galasso O, Ranuccio F, Gasparini G (2013) Midterm results and complications after minimally invasive distal metatarsal osteotomy for treatment of hallux valgus. Foot Ankle Int 34:969-977

29. Bauer T, de Lavigne C, Biau D, De Prado M, Isham S, Laffenétre $O$ (2009) Percutaneous hallux valgus surgery: a prospective multicenter study of 189 cases. Orthop Clin North Am 40:505-514

30. Bauer T, Biau D, Lortat-Jacob A, Hardy P (2010) Percutaneous hallux valgus correction using the Reverdin-Isham osteotomy. Orthop Traumatol Surg Res 96:407-416

31. Radwan YA, Mansour AM (2012) Percutaneous distal metatarsal osteotomy versus distal chevron osteotomy for correction of mild- to-moderate hallux valgus deformity. Arch Orthop Trauma Surg 132: 1539-1546

32. Roth A, Kohlmaier W, Tschauner C (1996) Surgery of the hallux. Distal metatarsal osteotomy (Bösch) percutaneous vs open (Kramer) procedures. Foot Ankle Surg 2:109-117

33. Maffulli N, Longo UG, Oliva F, Denaro V, Coppola C (2009) Bosch osteotomy and scarf osteotomy for hallux valgus 
correction. Orthop Clin North Am 40:515524

34. Giannini S, Cavallo M, Faldini C, Luciani D, Vannini F (2013) The SERI distal metatarsal osteotomy and Scarf osteotomy provide similar correction of hallux valgus. Clin Orthop Relat Res 471:2305-2311

35. Enan A, Abo-Hegy M, Seif H (2010) Early results of distal metatarsal osteotomy through minimally invasive approach for mild-to- moderate hallux valgus. Acta Orthop Belg 76:526-535

36. Scala A, Vendettuoli D (2013) Modified minimal incision subcapital osteotomy for hallux valgus correction. Foot Ankle Spec 6:65-72

37. Sun WD, Wen JM, Hu HW, Sun YS, Sang ZC, Jiang KW, Liang Z, Cheng T, Lin XX, Wu XB, Dai HL (2010) Long term efficacy of minimal incision osteotomy for hallux abducto valgus. Orthop Surg 2:223-228

38. Kadakia AR, Smerek JP, Myerson MS (2007) Radiographic results after percutaneous distal metatarsal osteotomy for correction of hal- lux valgus deformity. Foot Ankle Int 28:355-360

39. Gadek A, Liszka H (2013) Mini-invasive mitchell-kramer method in the operative treatment of hallux valgus deformity. Foot Ankle Int 34: 865-869
40. Martínez-Nova A, Sánchez-Rodríguez R, Leal-Muro A, Pedrera-Zamorano JD (2011) Dynamic plantar pressure analysis and midterm outcomes in percutaneous correction for mild hallux valgus. J Orthop Res 29:1700-1706

41. Sanna P, Ruiu GA (2005) Percutaneous distal osteotomy of the first metatarsal (PDO) for the surgical treatment of hallux valgus. Chir Organi Mov 90:365-369

42. Steinböck G, Leder K (1988) The Akin-New method for surgery of hallux valgus. 1-year results of a covered surgical method. $Z$ Orthop Ihre Grenzgeb 126:420-424

43. Weinberger $B H$, Fulp JM, Falstrom $P$, Anavian RR, Gore Al, Bazak I (1991) Retrospective evaluation of percutaneous bunionectomies and distal osteotomies without internal fixation. Clin Podiatr Med Surg 8: 111-136

44. Magnan B, Samaila E, Viola G, Bartolozzi P (2008) Minimally invasive retrocapital osteotomy of the first metatarsal in hallux valgus deformity. Oper Orthop Traumatol 20:89-96

45. Siclari A, Decantis V (2009) Arthroscopic lateral release and percu- taneous distal osteotomy for hallux valgus: a preliminary report. Foot Ankle Int 30:675-679 